\title{
Is gene discovery research or diagnosis?
}

\author{
Mark E. Samuels, $P h D^{1}$, Andrew Orr, $M D^{2}$, Duane L. Guernsey, PhD ${ }^{3}$, Kent Dooley, PhD ${ }^{4}$, Christie Riddell, $P h D^{4}$, \\ Kathy Hodgkinson, MSc ${ }^{5}$, Mark Ludman, $\mathrm{MD}^{6}$, and Daryl Pullman, $\mathrm{PhD}^{7}$
}

\begin{abstract}
The criteria that distinguish human genetic research from clinical molecular diagnosis are frequently practical rather than theoretical. They are driven by the availability and costs of the relevant technologies and the systemic level of scientific fluency in interpreting laboratory results. The guiding principle in the practice of medicine is the primacy of patient care. In the service of this overarching goal the defining characteristic of clinical diagnosis is the definition of the disease entity, even when no immediate treatment is possible. For heritable disorders caused by single-gene defects, identifying the putative causal variant is the goal of molecular diagnostics. Current technologies, costs, and standards of institutional infrastructure have not typically permitted novel gene discovery to be performed within the realm of the clinical laboratory. Discovery is usually funded by self-defined research organizations and carried out by self-defined research personnel with the primary intent of publishing findings in research journals. However, exponential improvements in technological capabilities and the concurrent decline in associated costs seem poised to recast this landscape, bringing to clinical medicine some activities now considered research. Even whole genome resequencing of individual patient DNA is within clinical reach in the foreseeable future. Genet Med 2008:10(6):385-390.
\end{abstract}

Key Words: genetics ethics, molecular diagnostics, genetics research, gene discovery, whole genome resequencing

Molecular genetic analysis in humans raises complex ethics issues which often impact significantly on the pace of genetic research programs. Research ethics boards that consider genetics proposals typically worry about privacy and discrimination, informed consent, who has the right to speak for the family with regard to participation in genetic research, the sharing or nonsharing of results, and the subsequent use of DNA for other studies. Yet analyses of genetic information are carried out routinely in the clinical diagnostic setting without direct oversight from an ethics board. Our goal is to review scenarios commonly observed in molecular diagnostic and genetic research settings, and to consider the two paradigms from both practical and ethics perspectives.

Research is defined as "a systematic investigation to establish facts, principles or generalizable knowledge." ${ }^{1}$ It is universally accepted that research involving human subjects must be

From the ${ }^{1}$ Department of Medicine, University of Montreal and Centre de Recherche $d u$ CHUM, Montreal, Quebec, Canada; Departments of ${ }^{2}$ Ophthalmology and Visual Sciences and ${ }^{3}$ Pathology, Dalhousie University, Halifax, Nova Scotia, Canada; ${ }^{4}$ Department of Pathology, IWK Health Center, Halifax, Nova Scotia, Canada; ${ }^{5}$ Discipline of Genetics, Memorial University, St. John's, Newfoundland, Canada; ${ }^{6}$ Department of Pediatrics and Maritime Medical Genetics Service, IWK Health Center, Halifax, Nova Scotia, Canada; and ${ }^{7}$ Department of Medical Ethics, Memorial University, St. John's, Newfoundland, Canada.

Mark E. Samuels, PhD, Centre de Recherche de l'Hôpital Ste-Justine, 3175, Côte SainteCatherine, A-733, Montreal, QC\#\#H3 T\#1C5, Canada.E-mail: mark.e.samuels@ umontreal.ca.

Disclosure: The authors declare no conflict of interest.

Submitted for publication November 2, 2007.

Accepted for publication February 20, 2008

DOI: 10.1097/GIM.0b013e3181770172 regulated and overseen by independent ethics review boards (REBs or IRBs). ${ }^{2}$ However, information about patients is also routinely gathered by the medical system and is often publicly communicated in the form of case reports that are not typically considered research. Thus, such reporting is generally exempt from previous ethics review.

The ambiguous distinction between research and practice was recognized already in the Belmont Report, the earliest codification in North America of ethical principles and guidelines for research involving human subjects. ${ }^{3}$ The authors of the report wanted to ensure, in part, that not every departure from standard or accepted practice with an untested or different intervention was ipso facto declared to be research and thus subject to research ethics review. At the same time it was important that major innovations were incorporated into formal research early on to determine their safety and efficacy. However, the authors of the report were thinking primarily of innovative procedures and devices, and did not anticipate fully the advent of the genetics era in which the "patient" can include other family members who may not currently be attending the clinic. At the same time the line between genetic research and practice is often indistinct as genetic research can produce results that are immediately relevant to the clinical management of a particular patient or family. In some cases the clinical management of a particular disease produces new information ascertained through patients who had never officially enrolled in a research study.

Bevan and Hardy ${ }^{4}$ state that "In general, we do not think of typical case reports as research requiring REB review, as case reports/case series are retrospective descriptions of a course of events that document some new aspect of care, the data are not 
collected systematically, there is no data analysis or testing of a hypothesis, and the work has already been done." Shevell ${ }^{5}$ suggests that "Case reports be conceptualized as a formulized description of a particular individual's history with a disease presentation and progress...to warrant publication, such observations must be in some way novel and serve to advance our understanding of the disease reported." Close reading of these quotations illustrates that the standard definitions of case reporting are somewhat uncertain regarding the boundary between clinical diagnosis and true research. Indeed it has been noted that the distinction between "research" and "diagnostic" genetics is often ambiguous. ${ }^{6}$ This may lead to confusion with regard to appropriate practice. Even the obligation to seek informed consent from patients before publishing case reports varies between institutions and medical journals. ${ }^{4}$

\section{THE PURVIEW OF DIAGNOSTIC GENETICS}

Scenarios of current genetic diagnostic testing include (1) screening for specific previously observed mutations in known genes, in new cases ascertained by nonmolecular (i.e., clinical) presentation; (2) screening for new mutations in known genes already proven to be causal for a clinical condition, in newly diagnosed cases; (3) screening for mutations in individuals genealogically related to cases already testing positive for a mutation in a specific gene, where such individuals might themselves be at risk; (4) screening by cytogenetic examination for individuals clinically ascertained with phenotypes matching those of known chromosomal abnormalities, or in some cases novel developmental disorders. Typically such testing is performed within a clinically certified hospital laboratory, or under the supervision of a certified molecular diagnostician. Results are communicated to the patient either by a clinical specialist or by a medical genetics team that may include genetic counseling. Informed consent may be sought from patients before molecular testing, depending on the exact nature of the tests to be performed. REBs play no role in this process.

Despite the tremendous increase in medical genetic knowledge over the past decades, molecular diagnostic laboratories regularly detect genetic variants whose interpretation is at least initially uncertain. These can include rare single nucleotide coding variants in specific genes such as $B R C A 1,7,8$ or novel cytogenetic abnormalities in complex pleiotropic developmental conditions, ${ }^{9-11}$ which are sometimes resolved by ascertainment of additional examples. ${ }^{12}$ These outcomes may be withheld or provided to the patient with appropriate counseling, with the understanding that the test outcomes are, at least for the time being, ambiguous. Diagnostic analysis may also be extended to family members in an attempt to determine whether the identified genetic variant segregates with the disease in additional individuals. In some cases family studies are critical to provide evidence for or against a genetic etiology. ${ }^{13}$ This defines one important distinction between molecular genetic and other forms of medical diagnosis, in that family studies (of multiple individuals) may be essential for proper diagnosis of individuals. This blurs the boundary between case study and research more particularly for genetic studies than for other types of clinical analysis. Published genetic case reports, with or without molecular results, often report family-based data rather than just those of individual patients (see selected examples ${ }^{14-18}$ ).

Recognizing this level of complexity, Parker et al. ${ }^{19}$ have noted that "an unambiguous distinction between clinical practice and research is impossible" for rare genetic disorders. They propose to define research primarily based on outreach beyond a "single family to the solicitation of affected but unrelated individuals." Although this criterion is nominally precise, scenarios arise in which it is difficult to carry out in practice. In populations known for strong founder effects, formally unrelated individuals are often genealogically closer than they themselves realize. Many examples exist of specific mutations occurring at a much higher frequency in some regions than others through founder sampling effects. ${ }^{20-22}$ It may be difficult to define the degree of relatedness of multiple affected individuals within a population, and hence the concept of "single family" becomes fuzzy. Extension of molecular diagnostics beyond nuclear families of probands may play a vital role in medical genetics practice depending on the local population structure. ${ }^{23}$

Even in cases of clearly unrelated families, the criterion of "solicitation of affected but unrelated individuals" seems excessively strict. Imagine two diagnostic laboratories working with families from very different populations, and independently identifying the same novel mutation in a gene under diagnostic analysis. If either laboratory alone is performing a clinical diagnosis, and if the laboratories communicate results to each other in an anonymized format, are they suddenly performing research? Such situations do arise in the research literature, ${ }^{24-26}$ and it is not clear that there is universal agreement on the distinction between genetic case reporting and genetic research in them. As with other case studies, clinical samples for genetic analysis have been collected and tested through a diagnostic laboratory, and results have been reported to the patient as part of the medical diagnosis. By extension, results may also have been reported to family members (defined in some fashion which may depend on local population structure). If patients voluntarily consent to allow publication of these results in medical research journals in the form of case reports, this would seem to obviate the need for REB review. It is difficult to ascertain from the wide literature on human genetics to what extent prior or post hoc REB approval is sought for such genetic case reporting, but it is certainly far from a universal practice.

\section{NOVEL GENETIC ANALYSIS}

Patients lacking any molecular evidence of mutation of a known gene have reached the end of the current clinical diagnostic process. Further molecular analysis has historically fallen squarely under the definition of research. In attempting to identify a novel causal genetic variant, the next step is typically a complete genome scan of patients plus associated family members, possibly with inclusion of multiple families exhibiting a similar clinical phenotype. For the past decade such genome scans have involved panels of polymorphic microsatellite repeat markers, commercially available and distributed 
across the entire genome at defined intervals. Recently, dense biallelic single nucleotide polymorphism (SNP) panels encompassing hundreds of thousands of individual markers have become commercially available and are beginning to be used for family-based linkage analysis. ${ }^{27-30}$ Following statistical linkage analysis, if a small chromosomal region is identified which shows evidence that it may encompass a causal variant, positional cloning ensues. In the past this has involved physical mapping of the relevant chromosomal region, determination of the protein-coding gene content in the region, and eventually mutation detection. With the advent of the Human Genome Project and steadily improving genomic annotations, this process has simplified significantly. Statistical analysis of mapping data are now usually followed directly by mutation detection. A number of methodologies exist for identifying the presence of variations in patient DNA samples indirectly, but the gold standard ultimately is always DNA sequencing for identification of single nucleotide or small insertion/deletion variants, or other molecular techniques for detection of larger rearrangements.

Novel gene discovery using current positional cloning methodology may cost on the order of $\$ 25-50,000$ per gene, depending on stochastic factors such as size of the family, nature of disease transmission (dominant versus recessive), occurrence of individual recombination events in families defining the size of a linked chromosomal interval containing the causal gene, reliability of phenotypic diagnosis (i.e., penetrance), gene content of linked chromosomal intervals, and timing of detection of actual causal variants (i.e., first gene sequenced versus twentieth gene sequenced). Such a level of expense and uncertainty is effectively intolerable to purely clinical budgets. Realistically, positionally cloned genes (approximately 2000 molecularly characterized monogenic disorders are currently curated in the Online Mendelian Inheritance in Man database http://www.ncbi.nlm.nih.gov/sites/entrez?db=OMIM) have been predominantly supported through public/government or private research funding agencies at the local, national or international level. Senior scientists managing these discovery projects may be $\mathrm{MDs}$, $\mathrm{PhDs}$ or $\mathrm{MD} / \mathrm{PhDs}$, generally in academic or corporate research institutions with molecular biology research laboratories and formalized publication milestones. In this context, taking account of the mandated goals of the individuals overseeing the work, the sources of funding, and the institutional homes, it seems clear that such activities as now performed represent research, not clinical diagnosis, and must be overseen by institutional REBs.

One area where there is already clear overlap between clinical diagnosis and research is in molecular cytogenetics. As noted above, cytogenetic examination of stained chromosomes by light microscopy represents a form of low resolution whole genome analysis already routinely practiced for clinical diagnosis, particularly in cases including mental retardation, developmental delay or syndromic developmental defects. ${ }^{13}$ It also presents an historical progression of steadily increasing resolution, using techniques with greater and greater sophistication. Beginning with whole chromosome aneuploidy (such as trisomy-21), karyotyping by chromosomal banding has identified numerous microscopically detectable chromosomal rearrangements ( $>3$ million base pairs) in the appropriately ascertained clinical patients. Karyotyping with resolution at the level of individual genes can be obtained using fluorescence in situ hybridization, however this method requires postulating a specific candidate gene or small chromosomal regions (as in Cri-du-chat, Williams, Prader-Willi, and Angelmann Syndromes and subtelomeric rearrangements, among others). Fluorescence in situ hybridization and lower resolution chromosomal banding fail to detect smaller chromosomal variations or variations outside defined candidate loci. Recently microarray technology has been brought to bear on this problem, especially with regard to changes in gene dosage or copy number (i.e., copy number variants $[\mathrm{CNVs}]$ ). The resolution of detection depends on technical details of the arrays used, but ranges between 1000 and 3 million base pairs. ${ }^{31-33}$ Some efforts have been made to develop explicit guidelines for clinical uptake of array-based molecular cytogenetics..$^{34,35}$ They currently suggest that whole genome analysis not be employed as a firstpass technology in cytogenetic analysis. However, they also note that the main issue is interpretation of findings, given that array methods routinely detect multiple CNVs even in patients without a clear medical ascertainment. To the extent that rearrangements and CNVs can be detected in individual patients with high explanatory value, the future will likely include whole genome ultra-high resolution karyotypic analysis as a diagnostic procedure in clinical genetics.

\section{NOVEL GENETIC ANALYSIS AS DIAGNOSIS}

The case may nevertheless be made that novel discovery work is intellectually more closely modeled as diagnosis rather than research, particularly where explicit intent exists to provide information to patients and families. For example, whole genome SNP mapping data, obtained while studying copy number variation, can also be analyzed statistically for linkage or homozygosity in related individuals or for linkage disequilibrium in founder populations. It is a straightforward extension that family based genome scans, and follow-up DNA sequencing, could potentially be performed completely in a diagnostic context, especially if laboratory costs continue to fall. The sharing of molecular data from multiple families, obtained in the same or different diagnostic laboratories, might in principle be no different than sharing of other diagnostic information. However, inasmuch as genetic information is often "familial" rather than "individual," and insofar as clinical genetic practice does not generally require prior REB approval, the standard of appropriate ethics oversight for clinical genetics activities requires ongoing consideration.

\section{MONOGENIC VERSUS “COMPLEX” GENETICS}

Distinctions must be made among different types of genetic analysis. High penetrance monogenic disorders whose causal genes are already known are routinely diagnosed (with or without molecular analysis) and reported to individual patients 
and their family members in a clinical context. The responsible clinician and the diagnostic laboratory anticipate from the beginning that molecular results will usually be interpretable, and are likely to be reported to the patient who presumably is known to desire such results (or would otherwise not have agreed to testing). Extension of such analyses to novel monogenic phenotypes seems straightforward. It is worth noting that $90 \%$ of the estimated 22,000 protein-coding genes in the human genome ${ }^{36}$ remain genetically uncharacterized, thus a large body of discovery work remains to be performed even for monogenic disorders. ${ }^{37}$ Nevertheless, emphasis in genetic research has shifted toward the study of complex, multifactorial diseases with oligogenic or polygenic inheritance and strong environmental modifiers. ${ }^{38}$ In studying the genetic component of such disorders, including for example obesity, diabetes, asthma, migraine, multiple sclerosis, depression, parkinsonism, etc., it is often envisioned that results will remain highly statistical in nature, and not routinely interpretable in terms of the genotypes of individuals. Although in theory participants could be provided with results in the form of probabilistic increases (or decreases) in risk for each illness, the medical value of this seems to be questionable for now. ${ }^{39}$ There is no clear path from the research results to a clinical molecular diagnosis in individual patients, as cautioned in a recent discussion of ethics of CNV studies in autism. ${ }^{40}$ For the moment at least, complex genetic analysis remains clearly in the realm of research (notwithstanding direct public access to commercial genotyping services providing dubious interpretations), although this too is likely to evolve over the coming decades as our understanding of the functional consequences of subtle low-penetrance genetic variants improves. ${ }^{41}$ Even for well-understood monogenic disorders such as phenylketonuria or cystic fibrosis, considerable variability in penetrance and phenotypic severity are documented. ${ }^{42-44}$ Despite the difficulties posed for diagnosis and clinical management by such observations, this has not led to questioning the value of clinical genetic testing for these diseases. In fact, there have been pioneering efforts toward population-based screening for large numbers of genetic metabolic disorders, ${ }^{45}$ many or all of which presumably have some degree of diagnostic complexity at the level of the individual patient. Such efforts have even been accompanied by cost-benefit analyses, pointing in favor of pre-emptive molecular diagnosis rather than symptom-based ascertainment for both metabolic and cancer predisposition diseases. ${ }^{46-49}$

\section{WHOLE GENOME RESEQUENCING}

A full discussion of whole genome resequencing of individual patients is beyond our current scope. It is highly significant though that one such study has already been published..$^{50}$ This study - clearly identified as research and not case reportingnonetheless reports sequence information for specific genes with known monogenic phenotypes (for example Huntington Disease, for which the patient is reported as normal). SNPs with previously reported statistical associations to traits such as risk-taking or cardiovascular disease susceptibility are also re- ported. Resequencing of a second full human genome has likewise just been reported, ${ }^{51}$ as has resequencing a flow-sorted purified X-chromosome. ${ }^{52}$ For the moment, the costs of whole genome resequencing remain excessive even for genomics research programs. However, costs have dropped precipitously over the past year and several companies are committed to bringing such costs within the range of even modest laboratories, and hence potentially within the range of clinical units. ${ }^{53,54}$ Implementation of such technologies is certain to impact medical diagnostics in the near future. ${ }^{55}$

\section{PATIENT RIGHTS}

As elements of genetic analysis mature from research toward diagnosis, a crucial question is that of guaranteeing patient rights. Who is the guarantor in scenarios outside the domain of research ethics board oversight? Genetics does not present insoluble problems in this area. Ethical behavior in medicine generally is assumed to be both possible and administrable, and is expected to be the standard of practice in all medical institutions, including those engaged in both clinical medicine and research. Obtaining biological materials for genetic analysis, use of those samples for defined clinical tests (that may ultimately include routine whole genome sequence analysis), reporting of results to patients and family, and public communication of results as case reports in legitimate medical journals with appropriate confidentiality, are already realized in current molecular diagnostic clinical practice. Extensions to novel gene discovery, at least for high penetrance monogenic disorders, seem incremental rather than substantive. McGuire et al. ${ }^{56}$ provide an interesting and cutting edge discussion of these issues with regard to genome sequencing in a predominantly research context.

The requirements for clinical use of patient materials differ from those of research use. This is equally a concern under current standards. The lack of consensus among research ethics boards for many issues in genetics ${ }^{57,58}$ is to some extent contrasted by more normalized guidelines in the molecular diagnostic setting. In the latter setting, quality control, sample tracking and data confidentiality are formally assessed and validated independently. The challenge is to provide appropriate ethics oversight of such clinical activities without succumbing to the bureaucratic burden of ethics review all too common in the research context.

In our experience there do not seem to be significant differences between the United States and Canada regarding the implementation and administration of clinical molecular diagnostics. However, we do note a potential difference in the context of genetics research, in that Canadian researchers seem to have greater freedom to transmit research results to patients, given that research results in general are usually not obtained in a Clinical Laboratory Improvement Amendment (CLIA)approved laboratory. The unavoidable limitations to quality control in research laboratories are (or should be) made clear to research participants in the process of obtaining informed consent. 
Indirectly related to the issue of patient rights is the question of reimbursement. Research costs are typically absorbed by the grants administered by research investigators, whereas clinical costs are managed through the health care system(s). Acknowledging that this is a significant area of concern, especially as expensive technologies such as whole genome arrays and even sequencing are brought into the clinical realm, it is beyond the scope of the current discussion to delve into the economics of molecular diagnostics in greater detail. The issue is similar to that for new medical devices and drugs, as discussed by Garber. ${ }^{59}$

Our personal experience is that patients with inherited disorders are profoundly impacted by the outcome of a definitive molecular diagnosis, generally positively. There is a wide range of patient interest and acceptance of the potential for molecular diagnosis. ${ }^{60}$ However, we believe that for high penetrance genetic disorders patients generally prefer to know such results, rather than to remain in a limbo of uncertain diagnosis. Patients may express relief simply if they perceive interest by the medical community in even establishing their diagnosis, particularly in the case of pleiotropic syndromes where even specialists without genetic training may find diagnosis difficult. The implementation of molecular analysis in a diagnostic setting has the potential to expedite such outcomes significantly, and an informal network of clinical molecular testing laboratories has been established covering many, though far from all, molecularly characterized monogenic disorders. ${ }^{61}$ The emotional and health care burdens to patients who lack definitive diagnosis while experimental protocols pass through funding searches and then extensive research ethics board review should be considered as major factors weighing in favor of extending the definition of diagnostics in molecular genetic analysis.

\section{CONCLUSIONS}

The problem we have attempted to address is whether the distinction between genetics research and genetics clinical diagnosis is philosophical and fixed, or operational and fluid. We believe the latter description is more accurate. We hope to encourage the health care and research systems to be more consistent and less severe with genetic research protocols than they often are, as they may not fully recognize the fundamental similarities between such research protocols and clinical tools already in routine use. Moreover, genetic research in some instances leads with high probability to a potential clinical molecular diagnosis. Although diagnosis is not itself the stated aim of research, in many genetics study paradigms the two are inseparable. In some cases the research may only have been possible with prior understanding that the patients and families would explicitly benefit from sharing of research findings, either via the research laboratory itself or through an associated clinical diagnostic unit. In these cases the incompletely defined route from research findings to the clinic is in fact a drawback, which might be best solved if the primary findings themselves could be made in a fully clinical context. We believe that some genetic discovery work, especially for disorders or syndromes most consistent with single gene etiology, might better serve the needs of patients if brought into the estab- lished realm of diagnostics. At a minimum, many aspects of molecular genetic work that are currently considered "research" clearly involve only modest and logical extensions of protocols which are currently considered "diagnostic." Some amount of intrinsic uncertainty in molecular diagnosis is already accepted, and accounted for in dealing with patients and their families within the context of trained genetic counseling units. Moreover the tremendous variability in responses of research ethics boards to genetics research proposals does not accurately reflect the enormous experience and consensus the clinical genetics community has accumulated in dealing with the outcomes of molecular genetic analysis in human patients.

All genetics attempts to relate phenotype to genotype, and the relationship between the research and the issue of clinical care rests in part upon the severity of the disease, the possibility of amelioration through treatment or screening, the penetrance of the gene and the recurrence risk to family members. Our key point is that the boundary between genetics research and clinical diagnosis is not only fundamentally blurry, but also moving. Despite this, in an attempt to place some clarity on the issue at least in the current context, we define a continuum of scenarios as follows:

1. Gene finding that is definitely related to clinical care includes diseases with high recurrence risk, high penetrance, significant morbidity and mortality and amelioration.

2. Gene finding that is possibly research/possibly clinical care: a. The determination of genetic variants that may alter (for better or worse) a disease of the type outlined above in which a major gene has already been found.

b. Genetic studies that deal with diseases with high morbidity and mortality, but for which the penetrance may be unknown and where the underlying genetic inheritance may be unknown, particularly those conditions with the possibility of amelioration.

c. Outcomes of whole genome resequencing protocols depending on the exact findings and the strength of phenotype/genotype correlation for a clinical diagnosis linked to a particular gene.

3. Gene finding that is definitely research:

a. Most whole genome association and CNV studies.

b. Anonymous population studies.

c. The search for genes that are quirky but have no known significant clinical ramifications, e.g., male pattern baldness.

For monogenic conditions, or diseases with significant burden and a genetic component, the ideal structure within which genetic research studies should occur is perhaps that of a clinical genetic register system. ${ }^{62}$

\section{ACKNOWLEDGMENTS}

M.E.S., A.O., M.L., and D.L.G. acknowledge funding support from Genome Canada, Genome Atlantic, the Capital District Health Authority, the IWK Health Center, Dalhousie University, the Nova Scotia Health Research Foundation, the Nova Scotia Research and Innovation Trust, and the Centre de Recherche du CHUM. D.P. and K.H. acknowledge support from Genome Can- 
ada and Genome Atlantic, NL Industry Research Innovation Fund, NL Department of Health and Community Services, St. Jude Medical, and the Janeway Children's Hospital Foundation.

\section{References}

1. Tri-Council. Ethical conduct for research involving humans. Canada: Public Works and Government Services, 2003.

2. World Medical Association Declaration of Helsinki: ethical principles for medical research involving human subjects, 1964.

3. The Belmont Report: ethical principles and guidelines for the protection of human subjects of research. US Department of Health Education, and Welfare, 1979.

4. Bevan JC, Hardy JF. Permission to publish case reports/case series. Can J Anaesth 2004;51:861-866.

5. Shevell M. The ethics of case reports. Paediatr Child Health 2004;9:83-84.

6. Pullman D, Hodgkinson K. Genetic knowledge and moral responsibility: ambiguity at the interface of genetic research and clinical practice. Clin Genet 2006;69:199-203.

7. Abkevich V, Zharkikh A, Deffenbaugh AM, Frank D, et al. Analysis of missense variation in human BRCA1 in the context of interspecific sequence variation. $\mathrm{J} \mathrm{Med}$ Genet 2004;41:492-507.

8. Phelan CM, Dapic V, Tice B, Favis R, et al. Classification of BRCA1 missense variants of unknown clinical significance. J Med Genet 2005;42:138-146.

9. Piovani G, Borsani G, Bertini V, Kalscheuer VM, et al. Unexpected identification of two interstitial deletions in a patient with a pericentric inversion of a chromosome 4 and an abnormal phenotype. Eur J Med Genet 2006;49:215-223.

10. Spruijt L, Engelen JJ, Bruinen-Smeijsters IP, Albrechts JC, et al. A patient with a de novo $15 \mathrm{q} 24 \mathrm{q} 26.1$ interstitial deletion, developmental delay, mild dysmorphism, and very blue irises. Am J Med Genet A 2004;129:312-315.

11. Pinson L, Perrin A, Plouzennec C, Parent P, et al. Detection of an unexpected subtelomeric $15 \mathrm{q} 26.2 \rightarrow$ qter deletion in a little girl: clinical and cytogenetic studies. Am J Med Genet A 2005;138:160-165.

12. Baynam G, Goldblatt J, Townshend S. A case of 3q29 microdeletion with novel features and a review of cytogenetically visible terminal $3 \mathrm{q}$ deletions. Clin Dysmorphol 2006;15:145-148.

13. Rauch A, Hoyer J, Guth S, Zweier C, et al. Diagnostic yield of various genetic approaches in patients with unexplained developmental delay or mental retardation. Am J Med Genet A 2006;140:2063-2074.

14. Tajsharghi H, Ohlsson M, Lindberg C, Oldfors A. Congenital myopathy with nemaline rods and cap structures caused by a mutation in the beta-tropomyosin gene (TPM2). Arch Neurol 2007;64:1334-1338.

15. Al-Dirbashi OY, Abu-Amero KK, Alswaid AF, Hoffmann GF, et al. LC-MS/MS determination of dibasic amino acids for the diagnosis of cystinuria. Application in a family affected by a novel splice-acceptor site mutation in the SLC7A9 gene. J Inherit Metab Dis 2007;30:611.

16. Shrimpton AE, Schelper RL, Linke RP, Hardy J, et al. A presenilin 1 mutation (L420R) in a family with early onset Alzheimer disease, seizures and cotton wool plaques, but not spastic paraparesis. Neuropathology 2007;27:228-232.

17. Rosello M, Monfort S, Orellana C, Oltra S, et al. [Subtelomeric deletion 9qter: definition of the syndrome and parental origin in 2 patients]. Med Clin (Barc) 2007;128:419-421.

18. Wajid M, Bazzi H, Rockey J, Lubetkin J, et al. Localized autosomal recessive hypotrichosis due to a frameshift mutation in the desmoglein 4 gene exhibits extensive phenotypic variability within a Pakistani family. J Invest Dermatol 2007;127:1779-1782.

19. Parker M, Ashcroft R, Wilkie AO, Kent A. Ethical review of research into rare genetic disorders. BMJ 2004;31 329:288-289.

20. Sheffield VC, Stone EM, Carmi R. Use of isolated inbred human populations for identification of disease genes. Trends Genet 1998;14:391-396.

21. Laberge AM, Michaud J, Richter A, Lemyre E, et al. Population history and its impact on medical genetics in Quebec. Clin Genet 2005;68:287-301.

22. Peltonen L, Jalanko A, Varilo T. Molecular genetics of the Finnish disease heritage. Hum Mol Genet 1999;8:1913-1923.

23. Hodgkinson KA, Parfrey PS, Bassett AS, Kupprion C, et al. The impact of implantable cardioverter-defibrillator therapy on survival in autosomal-dominant arrhythmogenic right ventricular cardiomyopathy (ARVD5). J Am Coll Cardiol 2005;45:400-408.

24. Leren TP. Mutations in the PCSK9 gene in Norwegian subjects with autosomal dominant hypercholesterolemia. Clin Genet 2004;65:419-422.

25. Timms KM, Wagner S, Samuels ME, et al. A mutation in PCSK9 causing autosomaldominant hypercholesterolemia in a Utah pedigree. Hum Genet 2004;1 14:349-353.

26. Zhang LL, Lin ZM, Ma ZH, Xu Z, et al. Mutation hotspots of SCN9A in primary erythermalgia. Br J Dermatol 2007;156:767-769.

27. Samuels ME, Dube MP. Linkage mapping. In: Encyclopedia of genetics, genomics, proteomics, and bioinformatics, part 2, Genetics, Canada: John Wiley \& Sons, 2005.

28. Samuels M, Higgins B, Provost S, Marcadier J, et al. New technologies in human genetic analysis. Am Biotechnol Lab 2007;25:22-27.
29. Matsuzaki H, Loi H, Dong S, Tsai YY, et al. Parallel genotyping of over 10,000 SNPs using a one-primer assay on a high-density oligonucleotide array. Genome Res 2004; $14: 414-425$

30. Sellick GS, Longman C, Tolmie J, Newbury-Ecob R, et al. Genomewide linkage searches for Mendelian disease loci can be efficiently conducted using high-density SNP genotyping arrays. Nucl Acids Res 2004;32:e164.

31. Feuk L, Marshall CR, Wintle RF, Scherer SW. Structural variants: changing the landscape of chromosomes and design of disease studies. Hum Mol Genet 2006;15 Spec No 1:R57-R66.

32. Redon R, Ishikawa S, Fitch KR, Feuk L, et al. Global variation in copy number in the human genome. Nature 2006;444:444-454.

33. Friedman JM, Baross A, Delaney AD, Ally A, et al. Oligonucleotide microarray analysis of genomic imbalance in children with mental retardation. Am J Hum Genet 2006;79:500-513.

34. Aradhya S, Cherry AM. Array-based comparative genomic hybridization: clinical contexts for targeted and whole-genome designs. Genet Med 2007;9:553-559.

35. Manning M, Hudgins L. Use of array-based technology in the practice of medical genetics. Genet Med 2007;9:650-653.

36. Clamp M, Fry B, Kamal M, Xie X, et al. Distinguishing protein-coding and noncoding genes in the human genome. Proc Natl Acad Sci USA 2007;104:19428-19433.

37. Antonarakis SE, Beckmann JS. Mendelian disorders deserve more attention. Nat Rev Genet 2006;7:277-282.

38. The International HapMap Project. Nature 2003;426:789-796.

39. Ropers HH. New perspectives for the elucidation of genetic disorders. Am J Hum Genet 2007;81:199-207.

40. Tabor HK, Cho MK. Ethical implications of array comparative genomic hybridization in complex phenotypes: points to consider in research. Genet Med 2007;9:626-631.

41. Kimmelman J. The post-human genome project mindset: race, reliability, and health care. Clin Genet 2006;70:427-432.

42. Kerem E, Rave-Harel N, Augarten A, Madgar I, et al. A cystic fibrosis transmembrane conductance regulator splice variant with partial penetrance associated with variable cystic fibrosis presentations. Am J Respir Crit Care Med 1997;155:1914-1920.

43. Mussaffi H, Prais D, Mei-Zahav M, Blau H. Cystic fibrosis mutations with widely variable phenotype: the D1152H example. Pediatr Pulmonol 2006;41:250-254

44. Scriver CR, Waters PJ. Monogenic traits are not simple: lessons from phenylketonuria. Trends Genet 1999;15:267-272.

45. Watson MS, Mann MY, Lloyd-Puryear M, Rinaldo P, et al. Newborn screening: toward a uniform screening panel and system-executive summary. Pediatrics 2006;117(5 pt 2):S296-S307.

46. Feuchtbaum L, Cunningham G. Economic evaluation of tandem mass spectrometry screening in California. Pediatrics 2006;117(5 pt 2):S280-S286.

47. Schoen EJ, Baker JC, Colby CJ, To TT. Cost-benefit analysis of universal tandem mass spectrometry for newborn screening. Pediatrics 2002;110:781-786.

48. Noorani HZ, Khan HN, Gallie BL, Detsky AS. Cost comparison of molecular versus conventional screening of relatives at risk for retinoblastoma. Am J Hum Genet 1996;59:301-307.

49. Joseph B, Shanmugam MP, Srinivasan MK, Kumaramanickavel G. Retinoblastoma: genetic testing versus conventional clinical screening in India. Mol Diagn 2004;8: 237-243.

50. Levy S, Sutton G, Ng PC, Feuk L, et al. The diploid genome sequence of an individual human. PLoS Biol 2007;5:e254.

51. Wheeler DA, Srinivasan M, Egholm M, Shen Y, et al. The complete genome of an individual by massively parallel DNA sequencing. Nature 2008;452:872-876.

52. Bentley D. The impact of new DNA sequencing technologies on human genetics. HUGO HGM2007. Montreal, 2007.

53. Bentley DR. Whole-genome re-sequencing. Curr Opin Genet Dev 2006;16:545-552.

54. Mardis ER. Anticipating the 1,000 dollar genome. Genome Biol 2006;7:112.

55. Robertson JA. The $\$ 1000$ genome: ethical and legal issues in whole genome sequencing of individuals. Am J Bioeth 2003;3:W-IF1.

56. McGuire AL, Caulfield T, Cho MK. Research ethics and the challenge of wholegenome sequencing. Nat Rev Genet 2008;9:152-156.

57. Glasziou P, Chalmers I. Ethics review roulette: what can we learn? BMJ 2004;328: 121-122.

58. Lewis JC, Tomkins S, Sampson JR. Ethical approval for research involving geographically dispersed subjects: unsuitability of the UK MREC/LREC system and relevance to uncommon genetic disorders. J Med Ethics 2001;27:347-351.

59. Garber AM. Cost-effectiveness and evidence evaluation as criteria for coverage pol icy. Health Aff (Millwood) 2004;Suppl Web Exclusives: W4-284-296.

60. Wexler A. Mapping fate. Crown, 1995.

61. Pagon RA. Gene tests: an online genetic information resource for health care providers. J Med Libr Assoc 2006;94:343-348.

62. Emery AE, Elliott D, Moores M, Smith C. A genetic register system (RAPID). J Med Genet 1974;11:145-151. 